\title{
LA ANATOMIA EN LA MEDICINA DEL ECUADOR
}

\author{
VIRGILIO ['AREDES I3OR, $3 A$
}

\begin{abstract}
"Sílo las tixuras caryadils de pasado están riags the pienvenir".
\end{abstract}

Alfohitr Reypts.

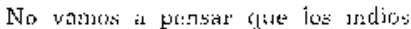
scualurianos prepizarriacos del ista-

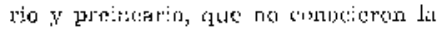
uscriature ni si aradu, les riada sti el hierro, $y$ rue pritcticiarul su Medicina de pensamiento mńficis $y$ senspirisirser desomar:izado, yropio de los pacblos plim:tivos, pulieron tenser esureimicnas de Anstomía I.Lumana, our necosi-

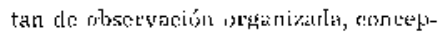

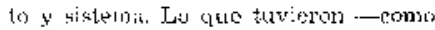
todo puldyly jrirritivo- fue su vocabutrio, easi desconucido hosta nuso-

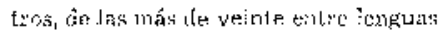
$y$ slialectos aborigent's ecuainrianos $y$ las nabidas de] guichur de los: Incas, vil)cubulario, este íltimos, para ilesjoftal vistered y extremidides del eucrpo humano: que no consitutasen una visarjizattión at conscimientos auatomicos $y$ que !o alcanzarion con da chservación desorsatrizade y parcia: nol enrmo husmasur, en da cuslit ptuitzriana con la

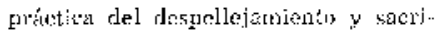

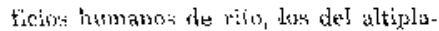
no en al précticu de eurar accidentes de frecipilación, [ric:talas y liersdas, que tambićn ocuparon a los de la sos. t:1, k misino que a lus de la selva oricalsal, juntos com les accistontex propios
LL Ju vidis en li1 selvit, gouereros y de «:izi:

Les lutis futeron embalsamadortac,

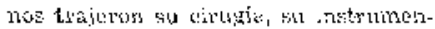
la\} puirúrg:co, nuevas pricticas retigesane con aicerilicios humanos $y$ st?

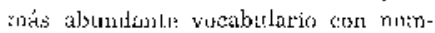

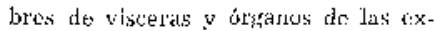
1remidades det sterpo humano, ยl una

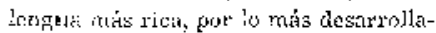
dii, comperada con nuesirus dialevtos abor f́gtł:es.

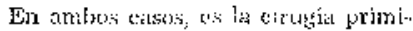
tiva la ifue llevó a iricas $y$ aborifgenes ecuatorianns al conocimienles pirvid: de liss partes det cuexpor bumano $y$ a darlar wh rumber en sus lenguas. Hi: hechicero sstuvo wás corca de eñle cunocimisnto cuado hacial empirismo

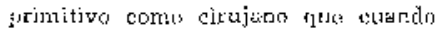
bacía pura hcchicería, lo mismo que tu nuestru tiompo, lis cirujanus viven nóts certa : te conocimierto anatónics) los sulanistas, juratue los primeros to iิextes snás a sil vista.

Espraja fuvo univetsidatcles descle la Bajj Ifdad Modia: Sulumatca en 1.200,

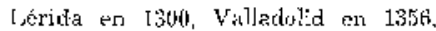
Huescat en 1354, Oslama ren 1449. 'Todás Lovieron su F'acultad de Mt:djejmą. 
In la Universidad de Vaicncia se esl.udir Anátomía HIurrana $y$ so particó disección dẹsde 1240. Cuando Muntpellier fue parte de di coronat de Arargón, en 1340), siz Esstatuto t,bizó dos diser:“iones por año a suls alumnos de da Horcultad de Medicina. A la Universidad de: Letrida se la aluturizó en 13211 para que obligue a sis doremtes a practicar diseceionens. Kin el sigho XV, los Reges Cutólizos exterdieron su autorización para hacer disecciones a todas Ias Facultades de Medictiná espanulas. Fintres los pajscs curspeos det ewos siglos, Fispaña anduru astc]antada on prácticas: de disceción y enseñutra de Anatomía Ilumana, dos medievs titulados en 5 us Facultades de Merdicinis tuvierum onasisin de disecar $y$ aprender lie mizteria coun dicentes hien preparadus, cuntondo - coint conlason- - con tratadistn.s que inicjitron une respeiable tradición er extudios de Anatomía Hurrana: a meditudss chol siglo XVI, Luis Iovera. dec Avila publicu en lutio su Artaturía, li primera aparerda en 厄spaña; por la mitad del mismu siglo, Bernardinu Monduña de Muesserrate publica la primera Aratomía THumana en lengiza española, y ya en la segunda mitar dol siglo apurece la Anatomí de Ju $J_{\text {an }} \mathrm{Val}$ verde de Anuzsco.

En el siglo de la fundación espanterle de Quitr, -.ol XVI- he aprendía Medirirna en Alealá de El1ares en rua1ro aiñs do estirdius; en la dtamada VIS[PEAS se explicand Anatomál. In todas kis universidaties españolas de exos fiempos se hicieron los ostndios siguiendo at Hipóerates; Galonor $\mathrm{y}$ u] strabisms, de Avicena, súlidamente ts. lablexidus como pensiniento médico.
Graienistru y arabismo dominaron la enseñarara pródica $y$ el ejercicio proier sionat, nati תu el galęrismo objetivo y racionalista de Gulteno, sizo el de las qule te êl hicieron, en continuirtad le pensuminento con !'a Alta Fdar Media,

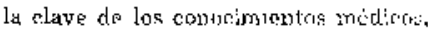

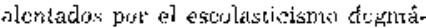
ticu glue así lo consagrú orlorpeciendo al adelusto riéd: [-3ajo Reyacimento, en los que dominó เ:L galeurismo dogrmátier: meneralizadoi, juntns com of intolectutifismo $y$ diulírlica del escolastitisino, quu sólo sirvió pura innedir nomo fuerzis poderosa e incontenible ed adclarto de la Medic:na. La manera detallada, minuciosid c imaginaiva de los árabes: tuvo 511 in-

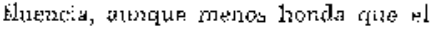
galen isinnt..

Fray que tener en cuerria alte th galenismo dogmático no sue un doño sc.. tolectual quo sufrió kólo España, sino tude la Ituropa culia. Til Renaciniento.

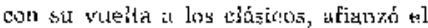
galenisino lejiss de abandonar]o, consiguiendu que se prolongue su dictachura intelectual por doce siglos, retrasando t:l avance de la Mericina. Las dicladuras intelestuales som rás durables y perriciosas para la rervovación del siaber, the lo que pueden elcanzar las ante ellas pobres y limitaldas dicteduras militares y polílicas ante ol gohbierno do Ins pueblos.

En Españu, el comotitriento $y$ enseñanza de Anatomia I tumana escátúu det gulenismo imperurste cr estrs tiesnpos. Montaña de inonsexrate y Valverde de Anstuse escribieron sus Aratomías saliérodose de Ja Anatomín galénica - de observación en gxan parto aninual. - y 
siguiendo el pensomielı1, vesaliano de La Anatomía observada y descrila son la disección: humana a la visia, alunque consorveuda sedimentos gulénicos, y es esta Anatomía vesaliutri y renacentis. ta, Jis quo instruyó con sus textus espaniules a los [urimeros médicess lloguclos a Quitu en el siglo XVI, que La aprendicron en jas universidiatés en las gue se titularon.

En 1593 estuvo casualmerile en Quinto el llatnado Doctor Dor .Juan del Castillo, que ni solieitó pereriso de ujercer ni se sube de sus titulus universitarios. Fue en 1.597 cuardu el Cabildo, Justicia y Fegimienta do Quito Lunotiú los títulus de Bachil]er, I,jecontiádo y Doetor iuncedidus por la tuiversidnd de Sovilia al Doctor Iron A1Furso Valdés, nanbrado Mlídier de ia Ciucterd en al misrou año, siendo el primer médico que homos podidu conocer con certeza que lifegó a Quitu, ciudart ell la que 1allexió corto tiempo despućs le su numbramiento. Fue él guien c4rnoció, vinn preparads en Espraña y 1.rajo a la Audiencia de Quito la Anetes" mía remacentista que gutió su práctica médica, lo mismo que los titudados médicos de la ciudad que lo sucelienor cum intervalos y en corlos ficmpos: los doctores Don Fexinando Menese y Mena de Valonzuela.

El 13 de abril de 1693, fecha memorable en ta historia de la enscñaniza médica en el Ecuador:, Fray Ignacio at Quesada y Fxay Bartolome García, $O$. $P$., fundar la FACULTAD DE MEDJCINA DE QUITO, con un Plan do Estudios te tres anos y dos catedráticos. En la llambinte laeultid de Quito se cormenzó enscìnando como etr Esipañit:

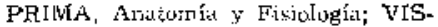
PFRAS, Paisligía; intrODO, Terapéutičs. No hube, como en Lspaña, proPesco destinsule exclusivamente a la Antomia, por exicasez de docentes - - $^{\prime}$ lo dus -. y por no habertos preparados en esta mitteria. Las chases se distaron, no comn en Espar̆a a la maJera veséliana - "puro observar y ver" tcl ORSERVATIO remacentista- sino (u) retrotedieron al CONSILIUM PRO y CONSII infeIsxtualistas, Leóricos y discursivos impuesils fur Ja filesofía escolástica l'uirante en Quitio, con su dogmatismo inedjevi].

Lia Anatoillía del Barroen, que continuó lá obra renacentista de Vesalio, con lis sistemátiona axtopción de la diseccisis del vadaver humaeso como única. [ridia sltu] comocintiento y enseñanza de ia Auatoryía zor Fabrizi da Aquapendente, nos vino (s) los dos pximeros bellemitess llegados a Quito en Jifo4 para hacersc cargo de la dirección del I SOSPITAL LE: SAN'A MISERICOR. DIA IEE NUESTIKO SENOR JESUCIRISTO, que lo haken des años despućs -..en 1706- cunndo estuvo en la cerpical de la Audiencia, Fray del Rosayio. Nuluramente que nos cstamos reîiriendo al campro de los conocimientos atuatomitus que guiaron a liss pricticas médicas y quirurgieas de Ios betlenilás del IIotpital. Los tres frailes beJermos urapen el pensamiento nédico deI 13atroco, cur Fabrizi du Aquapendente

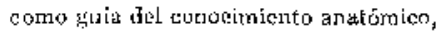
世 la por nusitros llarnatla CUARTA EPOCA de nuestros contocimientos médit:ss: la de la medieina inglesa de $\mathbf{S y}-$ dewhang y la Medir:ina sajona do Bu. 
herhasve, IJoffmanu, Và lelmnnf $y$ Van Swisten con is Fisintela Primitiva de Vienal.

Fin masa de 1736 llegan a $Q u$ lo, com la Misión Franeusa encargado do medit ut areo de merisliano en lá línea exuatoríal, e! méctico doctor esossep Jusies y el Cijujanor de dat Misión Dustor dean Serriergacs, traycada lus conucimiencos anulómicos adquiriclos en las universidades frajousts, los r[⿷] fin del Baxroen, ou rue se cnseñó Anatomía frụte al cadúres y se obligó a los alumurs a diserat. Seniergues cirujarn- estuvo más cerou de los conocimientus atsatómicos, operó es Qu:tir y Cuencil, lo vierur irtervenir xills colegas do armbas ciudados y com él esinvieron en juntas médicus. Hubu difusión de conucimientos an atérricos por intermediu de las relaciones profenionaless.

Se lizo sen Quito iu primera autupsiit en 17d6, según rusis intimman Areos y Samaniegu en l.A REDDTCINA EN HI, ECLADOR Y CRONOLOGIA MHDICA FCUATOIIAXA. Gustuvo Cevellis $A$, en su FVOTUCION TD. TA ENSFEANZA DE ANATOMML EN I. $\Lambda$ UNTVERSIDAD CENTR A.I,

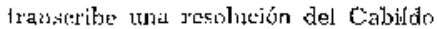
de Quito fechada en 9 de oetubre de]

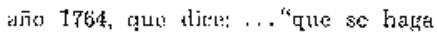
Anatomía del primer cuespon de difunto que hubiero muer fo en dicho honpif.al $\mathrm{K}$, turado del tilanc aceideste quc la molestado $\%$ aniquilado ereeridu número de gentes". Los diputudos designados por el Cabilda para ajecutoriar da resolución fucrun Drsm bisgo Doniso de la Carrera y el Alfótur Reisl Dion

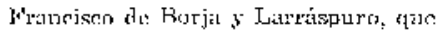

I, hivituon cumplir eno dos méticos a] 12 de wetubre de ese añn.

pira Carlos III de Jspeña un motialca de cultuma francesa - la de 1a Tluscración y los enciclepedistas .... Fin su

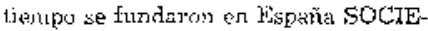
DADES FIIMN'J'ROPICAS Y F'ItoTHCNICAS destinadas a cultivar $y$ propagas el ILUMINISMO, tanto en

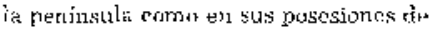
Aitúriśr, à doncle vinierou las maves guipuzcontan cun libros y publisurejtnes de los cnciclojediatas trascescs $y$ sus scguitdores y adniradores hispanes. F'ue el punto de arranque para do conociniento del perusemiente político de la época y la iniciación en las icleas emancipadoras, al ponerse al díc con las ideas de gobertar a los puetrless, la cienci:ir y la flosolía que ocupabeln a ios [ranceses e :nunietaban a Ios esprannoles esitudiosus. Por orden del gobicruo es. pañol al .'residente Don Luls Muñoz

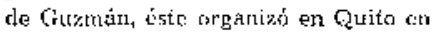
1791 la primera SOCIEDAD I'ATRIOTICA DE AWICOS DEL PAIS, COn Essejo como Secretarín.

Fintregado is sit cuitura, Carlos III exputse z los jesuíla: en 176\%; queda: do cle hechos sisprimida la RESL Y PON'IFICIA UNIVERSIINAI) DE SAN GREGORIO EL MAGNO de lo: padres de la Compañia y subsjsizond, en Quito una sola Universidad, Ial Real de Sunto Tomás de Acluiro de la Or. dist de Predicadores, la misma que fue

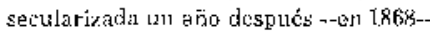
pur Auto Roal que debia sjecutorjar una Junta lianedal dic AYLICACIOVISS $Y$ TEMPORATIDADLS, ta misma que siambró a ins Decdores Dom klolchor Rivadencira y Jor Pedrn Olui- 
ťónc\% Cicnfucgens, catedrat:cis de la Universidad, fara upue redacten sus CONSIITUCION LS, quo diren: ". . . . pero por untablar un método provechsis, representa la nucesidad de dos Catedras de Medicina, ]a una yeura Instituciones on la yue los autores iraen competuots rarón de Anatomía y la otra para el mostodo..." Luege señalan el orden y los autores que deben adontátrse en la Ficultad de Medicina: "Esta será explicar prirrero la Anatomía por 1.oremzn Matster, Marlín MIartínez: AnAl'tu I aurecio, Vesalio o Bartoldino ejercitando desde el prinupio la $\Lambda$ ua Lumía Conprarada o disectión de los

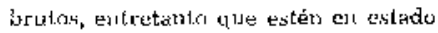
le ejercitar la propiu". Y añaden: " $\ldots y$ haran que Is den de menturia los $\Lambda$ forismos de Hipúcrates, a que seguirári les Instituciones Médicus de Boherhatave, con las notas do Alberto Inaller. EL ejercjicio será coun los denás, por preguntas, definiciones y argumentes".

1) la lectura de Ias CON'S'InTUCioNES, vigerdes tlesde I787 an que fute. min aprolaras por la torone, podemos damin cuenta de que la didácliea, plan de cstudios y directión de la cnseñanza médion y de Andtomía no había variaclo desde que se furdó la Facultad; lia lecturi clel texto pur cl Profesor, qu.? los xhlumus tenian que xupiar, in misn!r que unci rile otrn enmenstatio que sé idigrytha inscrtar el "magister", di que natio st atrevía a nbjetaxi el copiala habia que aprenderlo de corrido paro pusar lis prucbas, nétodo que hizo escuela, con sus cuizores hasta

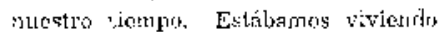
en la miład de la cultura dol lLUMTNISMO - Lan cerre a Cartus III y whs snteplcetuales consejeros afrancesados-. $v$ lis CONSTITLCIONl's señalan el ciercicio en lá "disección de los brutos" de La Ardurnín galónica medieval, pers a] misno ticr:po señalai como textos de comsulta a Vesalio --veliniéndrase a] HLTMANUS CORPORIS FAIBICA, fle $V_{\text {cosindius-- com el que ke abre el }}$

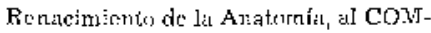
PENIDTO DE ANATOMIA de Lorenzo IIe istor, Profesos do ]a materia en All.1) (Alemania), quc vivió de 1683 a 1758, texto de Anaturíta del Barroco

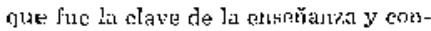
su1ta entre nosotros durante un dilufady tiempo, a li $\mathrm{NNATOMIA}$ COMPIAETA LEL HOMBRE $;$. Con todos los hallargyos, nuevas ductrinas $y$ observaciunes raras. IIasta ticmpo presente. $Y$ muchus arrertencias necesrias para la Cirugía. Scớn métcıdo cot une se explical en nuestro Tratro de Mastrid. Por el Ductor Martín Murti. леу ... Madrid 1718, auter espeñol dỉcl Bâtruen y el más drstatado tratadista de la Anátomía de sil tiempo, de la que se conservabit un ejomplar - que In comertames on 1943- entre Ios libros te la Cátedra de Anatomín do la Fatuiltad de Mitedicina de Quito, ton su cubierta de pergemino, con brochns y paistilres do lo mismo, sus primoro-

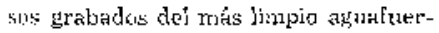
11: y la más pura trarieción renaccriasti. Poriblemetrto, pertencció a la $\mathrm{Hi}$ blistrea de la Univarided de San Guen gorio e Melsno y: fle al purar et la umiver'sitaria de li, Central. Con estos textus soñalidos para la enscräı\%d de la Anatomia, ]us CONŚll'tuCIONI's matidu seguir el grilichismo medieval y un Analomía el Barloco, petco ya es. 
tuvimos viviendo las segunda mitad de la chlturu de la liustreteión. Lu de las

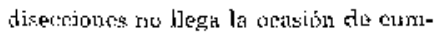
plirse, ni "cos los brutos", ni com "la propia", de que nos hablan las CONSTILLCIONES. Se siguiú haciondo la táchl, cónuda y perniciosa $M$ natumia Téricie cun la lectura del texto enpiadir y retención del snismo "al pie de la 'ctra".

A pedidos de Prewidente Don Tuis Muñoz de Gusmán; cl Obispor de Qur-

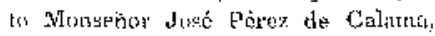
afticionarto a la Medicina y autor de? upisculo titulado DISEYTACION SOBRF. FI . IRENIKLIO DLL GALICO EN SL USO DE YAS LAGARTIJAS, prO. ycrto jnnuvactiones on los estudirs unjversitarios $y$ de Medieina com su PIAN, UTIL $X$ F'ACIL Y AGRADKIBLE DE LOS LSIUDTOS Y CATEDNAS QUH CON VIENE PONER EN IH.) ERICICIO DESDE J'L PROXINO CURSO DE 1791 .... ITHZ EN IA A REA L. L:NIVEIRSIDAD III SANTO TOAAS LE AQUTINO DE FSTA CIUTAD DE QCITO, publicadn el 20 do septiembre de 17\%l. E\} Plar clecia que su objetj. vo ora el do Jlegar a simplifitrar los es-

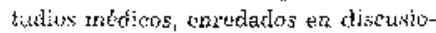
tes teulógicsis, teleológicas y clugmátitàs, olvidaudo la observación de lá nusuralexa, puro nlvidó señulis las materìs. pricticas y métodos para conseguir tian oporianos como atinados projuisitos. limitáladose a enunejas para Beodicina: "Me parece basla yor ahora una Cátedra de Modicuna; y asi que cornlinúe er su exercisio el dortor Berrardo Delgado", dcjándonos, eso si, un Fiel cuadro de cual fue el criterits con it que so enseñó ivadicto en la Fa:"r]- tact de Quito en .179], cuando habíats pasido híns de que Espejo esiturs tin tulado $y$ hirrinudo y sólo faltaban cualeo para su ruturte.

En 1787 presentaba Espejo sas l2EFEX:XIONES. Hay una severa (ritica de lit enseñunzis métlica de su tienpor. Recorrienda modificar el profesoradı con catedráticos traf́los de Maririd, rambiar los métodos lénicus de enscn̈anzâ: explicur Anatomia con eriterio whjetno, hacer disecciones. Aluba las Muatomias ya xiladas de Lorenzo Hei;terr esar láminas ele Cowpor y de de Marlín Malíne\%, siento su ilusracién andtómica de fluentes del barroes sajón y ispañol.

Scsćn afirma el Doctor Fuderito González Suárex cu su lisistoria Coneris! del Earlador en 1800 la J'aelzlad de sucdicina de euirn esluvo eerracha, por tríl la dre altumiss, resolviéndose aue los chrosus duxen cuatro añux $y$ e: e] cuaito zo setudie Anatrmía, on ol ya "itad" tuxto del Barroco de Lerenzo Feister de que nos haljlabun las COrs. TITCCIONTS de 2917, las intsires sue con buen juictı señalaban el estudis che esa usignatur"d ej el primer ario - llamado INSTITUCIONISS - minz:tras que en la reformil del play de esludios do troco antos tospues se mannta exindiar el abecedario de la Medicine,

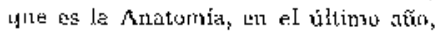
cs decir' que se tarminaban $]_{1>3}$ ex\{lzdios métlés's por doryde xacisnalment: debun comonzàr, urad isvercisón del urden Jógivo de asigniturus médicens que tanbién comenzó haciendo esueta, re. fasta, jero amorosalticule conservilda hasta nupalrus dín.

Desde 1809 liatsto 18.66 nadie se anta- 
clúa em la Feareulead do Medisina dr Quito. Desdo 1816 hasta 1827, lus testir dios, entre suspentidos o intervurip.dus, jo gue realmentc estuviares es maltratridıs y acabadus, "eun el descrcley a que esiaba reducida la Universidad sit fondess. eztatuteis, planes de cotudiv, sin lomalietad alguma:" no dijo el Conde Ruiz do Castille en commicación de 18.1 al Rector; $\mathrm{s}^{-}$ fuación çuc aleazzó toda la época de las campañas de enuancipación y se prolongri hasta 1827, acabando toun ki ensesturara, de escuela primaria a uлjversidal.

El 18 de marzo de 1826 se treal ía UNIVEIRSIDAD CENIRAL POS leq dol Cungresu de Cundinamarea $y$ el 26 de aetubre de 1827 se mganira da FACUI,TAJ MEDICA DE S,A TNI. VERSIUAD CLNTRAL, Un seis an̄ıIs te estudios. Annitomia Generui y Des-

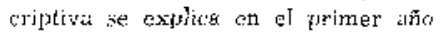
y para ingrestr a la fucultud stanculourbiaßu no se exjge gran cosa: הaber tecr y escribir, Gramátion Castel]ina y Aritmética. Como her ha nuesilos tiemjuss, sólo so babia cembiado cle plán de ratudios con espect trelala: alsín Jnoder. nizante $y$ demiagógico, pers la mentc del gue elseña: Ja uniciad $y$ ospiritu

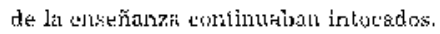
Lit 16 de nuvicubre de 1827 es nombraclo Profesor do Anatomia [ieneral y Parlicular el Doctor Ton José Arsturiu Natzanil. $N_{1}$ se lacen experar las reformets, porffide paxis reformistas do la enseñanza utiversitarja fuimos $y$ stomos nuy listox: é I Jecreto Tegisislativo de te noviombre de $1833 \ldots-y \leq 4$ en la Rrpublista.-. establece en Medicinil cítedras de Anatomia Genc'sal, एarkiculas

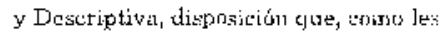
hat pasado a gran parte de nucstras ri: formas de la eriseñuma ruédica, se enuncia pero nos se umple, y e] misnti) Doctor Majzina liene due verue en el casso de tambión encaryarse de las cátedras de Terapéulica, l'yrmacij 'Teo. ricél y Prácficu, con el comsabida des-

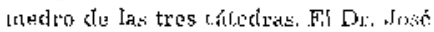
Antonio Mirzatua, nomiturado pitra explitar Amaimúa Gentral, Jadicular y Descripliva comrs asigrultura Hijarte pero que huva que dictuc ontas livera lo lo dispucstu- lue el protuel J"lafesor de Shatomía coms materia źmi-

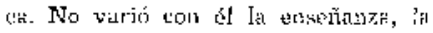
clase se rodujir a la lectura del ya thi-

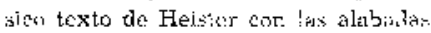
liminatio do Cowper, tal comó té apren-

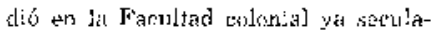
rizada the la Universidad Pública do Sant.u Tomás do Aruime. Nunca se h:\# tura desección ni un demostración frente il cadhver. Jel Doetur Marzasa siilo sabems que nacícó eis la segunda m:thr xel sigho XVII, estudió en lia Fusultad dis Melticina de la Univionirad Pública d: Santo Tomás de Anuiroo, en lat que recibio invealithura 1803. kin 1811 ejerúo on Aubsic, “. . "suffiendo in fracass en fa curación te un herido, pues se le acusa de haber osasionado la muerto...", emperián-

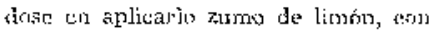
tursta consinncia que hast:a dejí uxis á lá herida. Jin 1827 lup designado Profeenr de Anatomía Generil, Parlicular y Doscriptiva de la Facula ad Métit:a do la Universidad Centuaj. Esluvo do Thisembro dh la Comisión de Botivas en 1830 y fue zustitilílu en lo Cáledra pos el 13atolor Vergara en 1835, 


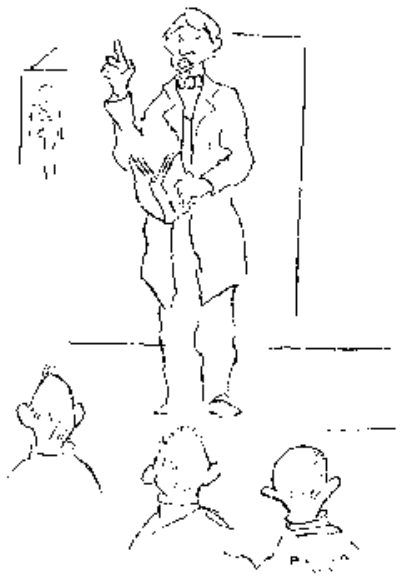

La prims:ra lección de Anatumix del Doctor Don Jusé Antonio Marzana (1827).

ui) sabemıs si pur fallecimienta.

Lu Legislaiura do 18 sas dió uno de los tantos tecretos reformistas upe hes mos padurito: cI I)ecreto Reformatorio de: la Ley y Regilamerters Givanoslrmbjanıs de 1826, que en su Art. 20 ..."ustabiece ru la Calase de Mediena Cácedras de Anatomia, Particular. $y$ Ijeseriptivia", yile conforme a trid ceión. no sin cumple por fatia de foudos. Lo do que los mombramiontos de profesores - gue sisupre se hicieron prot amisLarles to intereses de grupo- debian:

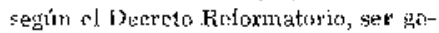
nados por concurso, tamporo se culrtjac, y cuandis hasta juccsirci tiempo so bs veitirlo ponicando en prástica, del roncurso se ha heclio laza larsa, mas incligna que el mismo lavoritismes que

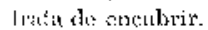

El Doctor Doin Miguel Vergara sl, acde a? Loetor Mär.anil el año do 1833 en la Cátcdra de Anatrmía. Sigue sas miä:ness métudios en la docencia, fuoro deje a]ço importante: la primera publi:caciót ecuaterianta que ha llegadir a suestro esnocimiento sobre Anatoníit Hunana: que fue la impresión de un CERTARHAN que tuvo lugar en Quit, e] so julio de 1837 sobre $\Lambda$ NATOMIA DESCRIPTIVA $Y$ SUS PARTES, OSTEOLOGIA, ARTICULACIONLS, SARCOLOGLA, MIOLOGIA, EEPLACXOLOGIA, ANGIO I.OGIA. NEUMOLOGIA, y DERVATOLOGIA. Se publibri bajo ol titule; de CERT'AMEN PUJIICO IDFDICADO A LA WIMGRI DF, ILUSTRE PATRICIO VICEATE LEON, HIJO DE LATACUNGA, QUU DESIJE LA CIUJ) $A D$ JYHL CLYCO NO HA OLVIDADO A SU PAIS NATAL, LEGANDO1.l' FONIDOS CON QUE SE ESTA. BLEZCA UÑ COHEGIO. Lmpresa en Gzuitu, $183 \vec{\jmath}$.

Los certánenes turversilarios lueron desde tirmpos coloniales una forma de armar espectículo, irnpresionando $y$ [alseandor los resultudo de lo enseñanzs. Nada bemeficiaron i In crseתanza y tuexon burmas de vanidad y brilo, livianus y sin aliances para estabiscer la vertad, eomo la "mesa redonde" y Congrescs Miédion de nuestras tiempos.

Jubemoy al Prusidente Don Vircnte Roratuerte el satublecimiente de less

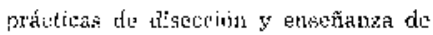
Austomia $x$ el calíver. Fn 1837 hidbiliró un lacal destinado a disecciones en el IIospital San Juan de Dius, con al nombre te ANFI'LA:RRO ANATO- 
MiflCo y el Docror Ion Miguel Versal'a de SOCIO PROPIETARIO JPF LA FACUISAD, o sea con su Cátc-

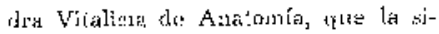
gujd ex]plitiondis $y$ chbligandes it sus

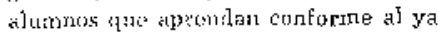
tan tonocitn texto de EIeistcr. Fin 1874 , Ettitenne Gayraud, nombrado Decuno 1 le ta Fucularl, hizo construír y torminar ese uño otro AXIITlATRO DE ANATOMIA, posiblemente en el misno lugir del adaptado en tiempos de Hoeafuerte, que ya estuvo inservible. Li 189t, ei ontunces Profesor de Ana. tumía fretor Ilat Joso Darío Echeverria, consiguio del Presidente Doctar I) An Anton:o Flures Jijón, la construc. ción de un muevo A.NEITEA'l'RO DI ANATOMLA, sibre el mismo sitio del sue teupó cl construido por Gayraud, pero sisuiendo Ia lánéa de fálorica del edificio del Hospital San Juan de: Dius, prueba de que los anteriores asl.11vierom returados do ella: anfiteatro de cierta amplitud para ese tiempon, las giae los illumunos no lisgeron a dier, que ya estuvo incómodio para 1915 , clidulo el Profesor Doctor Lais (1. Dávila lo hizo ampliar con urá xuteva ala que diaba a la calle Kusraies. Para 1935 et ctificio csiuvo ai dcrumbarse. Se expituba en una pequetia sili con escasas y desvencijadas boncas en la gue recibian clase más de cien alimmos, ba mayor parte de pie. La sala de disección ers un harcinamiento do malolientes jientas, la mayorif the ulumus disecaban en ios patios subre trozosi de labla $y$ ahí nijsumo resibían mudehas veces sus clases, por estar ocupada por otrir prulesor la única sala de clises disponible. El depósito de cadóveros era un moulón de descchos humanus rojdos !n, las ratas, h'latce enjambres de mo; t:ax $y$ un hedıx ilssupurtable. Algo maabas $y$ dienigritute que todos acepta bân rue no debsera whbsistir cortus d? pendencie univesituria, pur, qua nadie se molsisaba en ayudar a remediar. Los que ensctiazur y apren lio.yor Amatorsia en esu spoca recusedall hor rorjzados las condiciones en que lo: hit:ieron y córtor putieron sopurlartas.

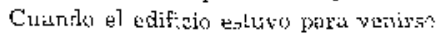
anceima co profesores y alumnot y obl:gar a thue so aterret: lik c] cises por tuerza mayor - -según informe de los arcuifectirs- - unica capiz de cambiur somojante estircio de cosas, con da generosit syuda de un Connté de Alumnus logramos cumvencer a las autoridades universitarias une sc hacia urgente la cumslrucetión blel aetual edificio del INSTITUT) Li: ANATOMIA de la ("al]e Sudiro, inaugurato cl 8 de octubre cie 19 šs.

Hon 1846 seguía el Joctor Vergara rle Protescar, con sus corlámenes. Publisó حon otros catedráticos- el de ANATOMIA, Quito. Lmpreso por M. Rivadeneira.

Continuatro en la docencia de Amatomía el minino lJoctor Vergara, el Presidente Jocaluerte legat a saber las aptitudes para lis discecón del alumno Juan the Acevedos $y$ to hace nombral DEMOSTRALOR ANATOHEICO, o sea un cliscutor: el primero que tuqin mos. A los atumnos del boctor Ver"rarix empezó jur primera ves a enseñarims amle una pioxid Imeparada pou él; estuvo hasta 1342 , aňo en çte se graduó. Fil Ministro del Intorior del Presidente Jon Vicente Ramún Roca 


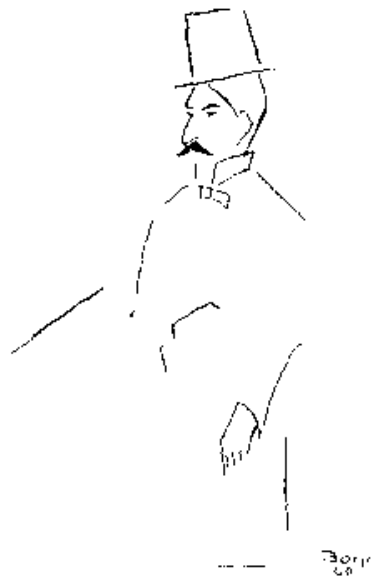

Dirckir Dont Sulan de Acevedo.

crea ja Cátedrat de Cirugía y lu mambra f'Tolesor en 1844, empcíñudose en

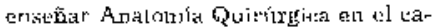
daver, funtersando la enseganzid de Anatomía cuardo estavo de Director de la Focu?tad desde 1847 y ntuvaruente desde 1855 on que estuvo de vuelta re E!tropa, it dunde fuc becado.

Fu 1846, cuando Acevedo ya no esluvo de Demnstrarlor Anaímilto, se of vid $b$ la práctisa de disección. bstudiaba Analomia el Doctol Don Tril'ael Ha-

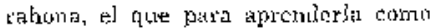
st debe, hizo de auloditiacía de Id dí aceción, ext:onclió cadávercs en urn apar1.adl, cuartuchn di:l IIospitas de San Juan de Jios. y ahi, com los unis mudimentarios justrumentos y en lo mikyor estrectrez c inconnoudidal se dió con alân a Ia disecrión, con los rețimnos $y$ amenazas judiciales de los deudos de! extinto anc tonía que esconder para sus jeráctícas. Pue umo de nuestros abundantes antodidact £.s.

Cuando el Dostor ditlienne Gayraud, traídn por cl Presidente Joctor García Motenm, fue nombrado an 1873 Decano de is Facullád de Mcdicina de Quj11 , elevo a sietc las asignatulas re $\mathrm{Mc}$ dicina, nu:nbranclo un projesor excluvivamente para Anatomía, que fue su eompanewe de labores doutur Dominiqus Jomes. Corro al doctur Vergara la susediera el señor disclor Camilo Cakires, que siguib olvidando las préc. ficas de diseceión del Demustrador Anatómico Acevedn, to :rismo que hizo su antecesur de cátedra, y we limitó a cambinr el texion de Heisler por ct do Boscusa. Gayraud projectó la edjiciccón de un ANFH'BATIO ZNATOMICO rue hizo rúpiditisente conslruír

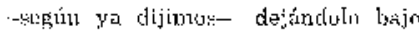
la dirección del Dysetor Dorrec y corsigtuiende tal mizyor ivant:e de nuestr"

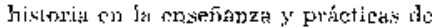
Anatmia. Con Drmec, Gayraud traju de París maturtal didáctico que aún so monserva, instrumental jara disección, wue lit slejarest establesida, moterial para conservación de radáneres In? ji:yección de furmol, técnica cute la intrordujerin por primera vez en el pais. Obligaran hacer précticus de disecétum a los alumonos. (iayraud nombró po: comeurso rale fue rigurosamentc cuir I)lido - thi si -... varios Ayudanles de Anatomía, un Prosector y defes de Trabujois Frácljous, que doji señulathos

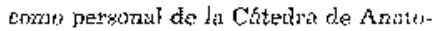
unja, ln más comngtotat, numerosa y bicn atcontida cle la racultar de stse tiempo. 
Se dietó claje fretate a la pjeza preparerla, consiguiendo la provisión de endáveres -. -upe nunca fallaron comes ahorn-- mediante an Dectetru Hjecu(.jvi) que exigia que debia ser inmediatamente allopsiads torcho endúver de Huspital $y$ servir a la Cáteóra, Dectetr iluc se cumplió tiolmente mientras vi-

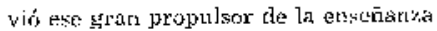
Médica on el Eevador cue fue ol Presidemle Uoctor Grareía Moneno. Después tle él, esta ıbliģación indispensiabje para la docencia de Anatumb́r cquedó dejinilivamente sepultada. Trajo Domec la escucia de anatónicus franPiesix, rom el TRATADO DF ANA'TOMIA GENERAI V DLSCRIPTIVA -por at Doctor [N. A. Fort -.. Catedrát:co Libre de Atuatumia ta París, adorrado rom 73 grabactos en el texta, versiót: wastultant sen murhas adiciones $y$ riritas de $A$. Séncbez Futritumantes, Madrid, 1871. Sucedió al Iroctor Doyne: uno de sus avestajadon discípulos,

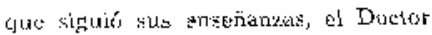
Don Antonio Fa'coní. En 1875, thentimces I icunciaclu José Dusio Echevestú: fundó una ESCUELA DE' $\triangle N A$ TOMIA - pl-ivada - aýludado por um grupo de sus condiscip̧ules, en la que so aptenklta dinectundo. Esc mismu ẫo, hicieron una EXPOSICION SM: 'PRA.

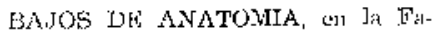
ealtat, la primeta de nuestra historia misicta; tuba un alto acadćmico cori lisrgns eliscurkos. En 1880 el dector Iidhererria abre en su casa nera lisCUEI.A PRACTICA DE ANATOWIA -con módicas pensiones. - según anuncia en la prestá. Nombrudo Protesor de Anatomía en $188 \%$, wirece al Decano wiganizar la su ya tercera ESCUHLA

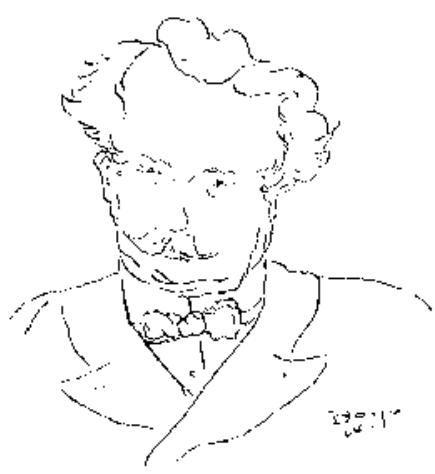

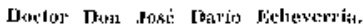

DE ANAROMIA - gratuita esta vorscrvidú con la culithoración de su! i alumness. Eno de los aciertos del sobierno del Destrir Caamaño fue el dis telebrar un contratu t:ula el Dortur Echevertio en 1888, pars que se porty di frente, ontre chas actividuluss, h. an CUIRSO DE ANATOUIA PPAC'TlCA, que reglandentó el gobierno. F': tul conld'itn lour scis años forzosos, ton un sueldo de tien pesos mensuales.

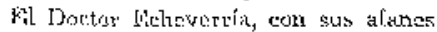
$y$ sus ESCUMEAS I)E ANATOMIA de 1.xdos los ratiecs, lue uno do los tenaces sultodes de la materia, de la disección y de la cuseñan\%e objetiva frente is la pioza preperrada. Continuó ja scuelid de anatormitos franceses do Ia copoca, truida por sh matestro Domec, con el TRATATOOJE ANATOITIA de Fort como texto de eriseñanza.

La FACULTAD DE' MEDICINA IDE; GUAYAQUIL se ubre el 7 de joviembre de 1877, con su Cútedra de Ansto- 
mía explicada por el Josctor Don Manucl des Tránsitu Pachecr, cirujano de frostisio qué exp!jó́ bbjetivazente e hizo disucciones la enseñunza médica comelzó en Cuentil desde 1868, pero su Fratlad ale Medicina no guedó deFinitivamente establecidis sino el $31 \mathrm{dc}$ rivembre de 1890, con su Cétodra de

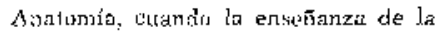

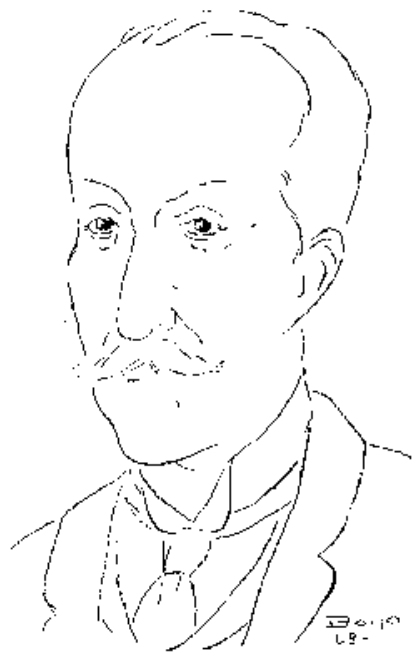

Dostur Dun Gillermo Oviónex.

mattrial ya había sido transformada en las dos Facultades que la procedieron, sé explicabr ante lá piozia y se clisecaba. IEl pensamiento del J'ss:tivismo Naturalista de la Murbomía de esos limpos se hubia exiablecitts definitivitmelate, ujanzándose cun los liberales en el purder, que teorganizan las l.te: utiversitades desde 1895 a J397.
En la de Guayayuil queda en Aneltowía o] misuro Doctor Pacheco y on la de Quito entra el Ductor Don Gixiller. mo Oxdóne\%, uno de Jos profusones do ialentu que han bonralo a ia Fasill. tal1 de Quito. Catcdráticu de gran es. tilo, se esineroi en explicar Anatimia General. Establecí la disertion entre lis aluminos, que nuevamente estuvo olvidúndoite. Hizo nombrar F'rimer y Segunto Ayutantes con funciones do Disector as. Siguió lá sacuela francesil, introrlusiendo en tol primer lustro de nuestro siglo fa primera eslición espaIrolit de lu Anatromín do Testut, modelo de literentura cientifica hasta hey no superada, que acalyaba de aparecer en

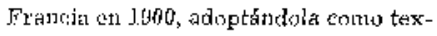
Lo. Mantuvo correspon rlencia cientifica (u) Tostul, gue lo citat on su Juonumental obra: al trilar de las varielstes te comsistencia del uvario.

Después del mağsteriu de Ordóntez en Quita y Pucliese en CHuáaguil, Jas cátedras de Anatomín de fas tres $\mathbf{F}_{\Re-}$ sultade do Mcdicina ecuatorianas sim givien el rumbo do los anatomistas de la esuele francess oncabezada por Teslul. El pemsiniento del Pusilivisisue Naturaliste, objetivo, metólico, realista y claro, ğuió la enseñanza. El conocisiniento de li materia por la labor de diseceión, la preparación de piczas axatómicas, In ennscrvación de cadiveres, cl orden, c:Iaridad y capaciclad deseriptiva de la exposición docente, el a'bandono de lo intelestudista y retóJico pur lo objetiwo y presente, ha siclo el Tesultado de la srientación de lí en. seríf]za de Anetonín on Lus Tacultodes de Medicina ecuatoxiande, con adelaviados como el Doctior Juan de Ace- 
vedis, dutordidactas del conocimiento anatomico cono el Doctur Ra!ael Bawhoma, ducentes laboriosos o jnfatig:1bies como el Doctor Jusé Darío Feheverriat, relultraduros, orientadoris $y$ construstores como el Dortor Disnituique Domed, dedicados y trgatizadorés cirno e: Dostor [MLnue] de! Tránsito Purbecu y desinleresadns valentos eomo e] del Dorcior Guillermo Ordónoy.

Las cátedras de Anatomín de las tres hacultades ecuacorianas fucron do Ias: mejor servidas y atendidas, se expl:caba cunfurme a Ia traficional estiela Jrancesa de su mejor época. IIoy: (wo: las obligadas desviaciones hacia lo nortedunericano, en nomenclatura, Lebidis a la próctica de la sledicina de inlluencía norteamerieana de ia época, los d]utmos reciben elase en innplitis tocales construítos para su función en Quila y Guayacuil; tienden al cumoc:miento príntico por la disección obligritoria y reglamentada, en sulas adecuadas; se han acloptado muchus de los modernos nedios de preparición de piezos anatomicas destinadas a lin demostración y explicación docente; hay Jej'es de Trabajos Prípticssi Ayudantes, Disteteres y Proparadorcs. El nú mero erecido de ulumnus ha vensto a dificultar labores y lis falta de una disposición zue ubligno a que todos las eadáveres de lus houpitales pasen por los Anfiteatros Anatómicos, como lo Гus: cri tiempos de Iromes, pouse su seríos compromisos a los docentes, eln. l.spectindo Ia enseñanza el forma alermante, por Ja carcncia de maturial d:dáptices. La salvaciól ostá en ensomtrar una nulera dispusicjón y un gobierno que la acoptc, on bien de la en- stuneny de Anánmíá.

No contamos en el Iricuador son uná BIBLIOTHCA DE PUBLICACIONFF I) MEDICINA NACIONAL, tampoe, hay UI INDICL BIHLIOGRAFIC:O. La laboriosa y ortenudir BILLIOGRA JiA CIFNTIFICA HCUATORIANA, del i]ustrado bib.jógrafo Caros Mennuel Idarrea, ro trae una secoción de Allatnmía. Nuestrus kiblictacas públicas y axivorsilarias no tienen seceiones rompilelas te aulores nacionales ui de materios naeionales trutudes por varius. 〔 1) más custuleto de publicariunes mídicas nacion:ales está en la Biblioteca Lutuatoriana de los jesuílas -en Cutocollan- y en la colección que fue del bibliofilo Ln. Carlos $A$. Rolando, doneda a Ia I3iblinlece Municipal do Gua-

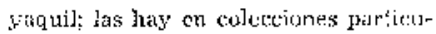
lares que pir su índile no son accesibles a todos. Ante la falta de tн insustitúbles ruedjos de infortuación, el estudio bibliográfico do las publicaeimes de Zledicina Narioma imposibilitan a quien lo intente: no pudiendo nosotros hacerilo simo en torma jtuperfecla, plagada de vacíos. A sabicudits de lo incompleto de nuestros dates, vayamos a la coumersción do lo que han publicuto sobre $\Lambda$ tuatemía IJumane los médicos ecuatorianos:

Moreno Tinajern, Dr. Manuel.-Conferenes a l'Eixternat des Hospiteux de Prujs. 3: Edition revue el augmentie: ANATOVIJ', 24 l'yscículos, paris, 1935.

Sastlanla, Dr. Antonio--Cuadros de Anatomía. V Libros. Trrojronáa do la Iriversidad Centeal: Quito, 1935-1942.

Areos, J $)_{1}$. Gualherlo.-Pintjpologia der habitunte del ali.iplano encuatoriouso. Impronta do la Lniversidad Centlit, 
Quite, 1938.

Santiarea, Dr. Alulonio,--Contribueiron al sostudio de la Anetomía. Andes rte la Unjversidad Cenlual. Non 30?. Iniprentin de la Liniversidad Centraí, wui10,1939 .

Suntiana, Dr. Antonio.-Los indius de Imbabura: sti cranenlogía. Anates: de lá Universidad Central, Nos. 314 y 315. Imprenda tie liz Universidad Con[Na], Quito, 1942.

Santiasa, Dr. Antonio y Paltán C.. Dr. José Desvid. -Contritución al eitudio de li Morfología ectatoriana. I.a dentadura de las indius de lmbabura y Chimboras. Anales de la tonivaridad Central, Nos, 314 y 315 , Guito, 1942 .

Santiuler, Dr, Artoridu y Pa]tár, Dr. Jusó Dawel. Contribución al ostudiu do fa Antropología reutoriana (dentadura de lia raza india). Trupresta de ta Universidad Cencla 1 Q Quito, 1942.

Santiana. Dr. Antonio: Conixibur:Lón ari astudio de la Anatumín del rọn cién macida (situls inverstas). Inlplenta flo [i] Liniversjad CentruI, Quít, 1942.

f.con Burja, Dr. Naujel.-Anatumía y Fisiohgia. Imprenta de la Universidad Centrat, Quilo, 1942,

Turriel, C.-El cuarpo humano. 1mprenta Talleres Mumicipales, Guayaguil, 1946.

Paltén, 13r. Josó David. -And1, smía TIumena General y Descrjptiva. AdapLadir a Ins.s neesitlades de médicos y nảunlólogise. T’ata nlaumos de primer eterso de Utiversidad. "3 volúmenes. Edicuria! Unziversitaria, Quito, I vel, $1961 ;$ IJ $y$ JlI vol. 1962 .

Yaltán, Dr. Jusé 1)avid.--Anatemía, H'iviósysta c Higiene. Editurín] La Sa- llde, Quito, 1959.

Surtiania, Dr. Antonen-Coritribución al cestuclio de les Antrupología seua-

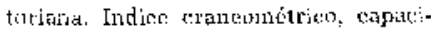

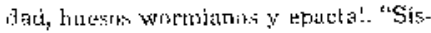
iole", Quito, 1936.

Torres Orclóñsz, Dr. Cruillermi.Apuntes de Émbrinlogín pary los titudiantes de Medicina. Imysenta Universiducl Centriel, Quilo, 1926.

Lripez Buca, Dr. Francisca-Languette J-Trpatirfe et lithiase biliare. I.yon, 1918.

La REVISTA DX LA FACUITAD DE CAENCIAS MEDLCAS (Fpoca Ĺnica). Innuenta de la Universidar Cenm \{:a], Quito, ha publicado:

Partes Borja, Virg!lio.- Nuevas chservacunos nobre el poso de las glán. dulus geritales del homble. Vol. IV. julin-licicmbre, 1953.

Cevallos A. Ijr. Gustavis,- Anatumía de la reggón hiputalánicá. Vol. IV, juliu-diciember, 1963.

Covullos A. Dr. Gustavo.-Fivolizión le la enseñanza de Anatomía cul la Universiad Central. Vul. Il, eneri)-junio, 1951.

Gaz'zón S. Dra. Bertha-Ooluservauimes sobre peso y t:upacidad del cisrilzim en el alliplano csuatorimo. Vol. VII, iLkilo-diciertbre, $\mathbf{1 9 5 6}$.

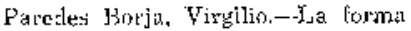
es Andonita descriptivia. Vol. VILl, julitr-di:itmbre, 1957.

Cevallos A., Dr. Gusiavo..-Síntesis de la srrigreión lincéticia, Vol. It, juliow disiexube, ,2952.

Cevallos A. Dr. Gustavo,-Andrés. Vosaljo "Mittir de la Anatomía". Vol. VIIL, anero-julio, 19;0).

Cevillos A., Dr. (iustavo.--Iuesos 
sexámoideos. Vol. III, abri]-julio, 1952.

Ceviallos A., Dr. Gustavo,-SarraliTatión de la quintel vértebra lumbar. Vol. IV, enero-junio, 195is.

Covallos A., Юr. Gustavu,- Evolicién de lia esrseñanza de Mriätumía en lat Urivisrsirlad Central. Vol. II, unel'ujunio, 1951.

Cevallos A., Dr. Gustavo.- -H:storia del Anditealu suatomieo de la liniversidad Central. Vol, YV, juljo-disiem. bre, 795 ?.

Paredes Eorja, Virgrilio.--Lat ctusebanfa do Anatomial. Vol. $V(j$, iujiarld:siembre, $195 \%$.

Paredes Dorja, Vurpilio,- Un tyatido de Anatoría del seteriestes ícosmentarios al libro cle Martin Martixez. anatómiço español). Revisti Médica, No ], abri], 1943. Impreuia de Ja Un:vessidad Cuntral, Quito.

Iua Aritomía ha sirlo eutro nosetros sioncia de escasiss cultores, qua han sebido con livikuír con sus datos a la Antropologia y ensễanza médica ner:ional ocupands noblemerte su tienpo en escribjr sobre una vastal disciplina guc necesita eonfrontaciones ]ol:aics, sobre todo en Mior rología y relacjones de órgenos, de bo que se conscrva (llyservaciones realizads por los alum:ks, recog:das ter algumos años, que aun गา han sido publicadas pur soso. tris. Hay observaciones dispersels pid-

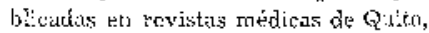
Gruayaquil y Cuenc:a, las qua: no uns la sióo poushle comserturilas.

Arida han dicho de la Anaturnía lus que la conocen superfecialmenit. No hay tiencjas átidas ni amables, huy rimeias bien explicadis y mal explicadas. Es el clocente el que haca a la riencia acougdora, con su arloc pre es un don feliz con el que se nace; la ilustración es ia raue so consiggue y amplia. [Tu buen prolesur de Anatcomía puosts scrto quipit engtá un don natural de enseñur, ac allicione a la ciencia $y$ lä ejercite; yuion tenga solo dedic:atur. af:enes $y$ conociniento, si carcoe dol arle do enseñar, llegará a poriconto de la momeria y wlverá áridid e insoportálble la materia.

Lia Arialomía nación on las atoligida Gzer:ia por uspiracion de los artistas y no por esfuorzus de cientriffieos mi de nudi-

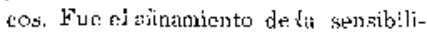
dad estética dé :dómiración al desinudid: lo que llevir â los primeros esludiess metodizados solure Anatomia Humand, for Leonatdo dä Vinci. La Anatomía ro es una cicncja acabatda, cumo lambick srecu bis que poet se han inter'esado en la discipaina. Ningura ciencia es acabuda, todus están en constonte rambio - no decimos progreso porqus no aremos en la teoría do] progresosivo en el perratuento muctar del conocmiento. Geteralizacisstes de concepto, mor Jolighta y relaciones de lus urgaurs son lus establecidos por las anatomías do las diferentes cseuelas, con rbservaciones $y$ estudios de sus tospect1vas zonas geográlitâs, gencrutizaviones que hay cue aplicarlas, the tomo wlave, sinu como pauti, oldenilción y metódica dẹl estudio de lis $i$ nal-

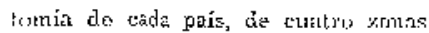

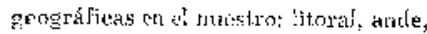
interande $y$ sclva oricustal. Para llesanr al conorimiento di la morfologín del hombre ecuatoriano, ton el que stos enpeinumus con nuestros alummos, ci campr de observatión os amplie; tu 


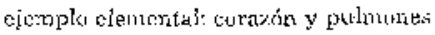
lieno:s pas'iscularidades definidats on ol litore's intorand? ezuaturiand: que tie-

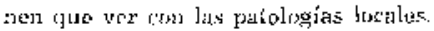
Fil at:in a fa llaneza y a lí simplifica-

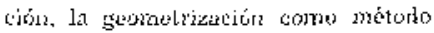
patra entender la morfolngégia, el orten y la limpridez del emeeptes, juritos con e) antitluginatisnu, geveralizador $y$ c]

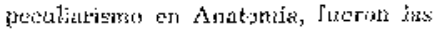
Ienctensiag ryue nos gaviarom.

Ni la enscràaıź de Anatucáá ...ni ninguna ensetianza. . requieren de sílo Lakroressidiad. Espiritu de observación - el "mantener siempre abiertek lia yupila", de Ollcga--, el saber juterpretizl. ron realisino lo rue se ve, a ta majeral genial de los racionailistas franceses, ja treación $y$ el vuclo inasinativo del aurabismo de su ćposa de grandera en los Carlifatos, neevitat el Prolesor de hratorita clue hace su docencia coun vocacion $y$ la dirige despertando afán y su-. siriendo tis la mento sel alummo la réstrlución de problonmis anatómicos, des. pertando st curjosidad, que lo conducirár al campo tio lá investigación, el cunucimianto desiuteresado o de apli. eistón en Tredicina.

(Dibujos (fol autor)

\section{TIBLCOGIRAFIA}

l. ALCDS, 3\%, GIAJdF!RTO. -Inii Methici-

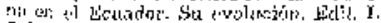

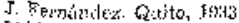

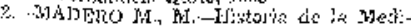

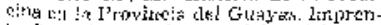

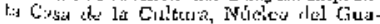

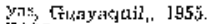

3.- SNALARILGO, J. 3. Cronalosta Médien Fontatn:iana. Eớit. Císa de la Cutterri Eryst.uria:1a. Q:rit. 14.57.

4.-MTONTUIRO CARIRON, Di. J. A. -Modicos $d x$ A,rer y de Huy. Vaines de 3

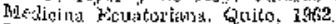

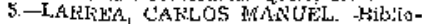

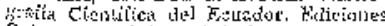

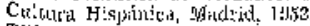

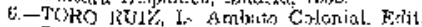
Tribunat. Ambato, $155 \%$.

7.- CEVALLOS A., Dx. CHUSTAVO.-.H. tor'i lel Anfíciatro Anaiónico dit le

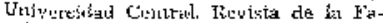
culati te Cieneias Médiens. Vol. IV: ju-

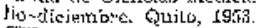

8 CEVALLOS A., Dr. GUSTA VO-Fulu-

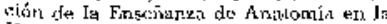

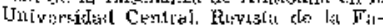
euliut re Cicncias Médieas. Fn[. II, enr:['u-junio. Qui1it, 1951.

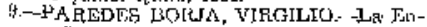
atriñuza de Anatamia. Revista de: ia Fin-

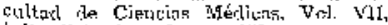
juJio-diciembre. Quito, .1956.

it--SAN'TSANA, Dr. AN'LONIO--Contribr-

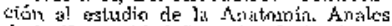
Ge la Uniwersidud Central, No 307. Quito, 1930 .

1.-MONZALYZ SUAKFZ. F.-Historin Giv-

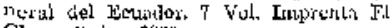
Clero. rizuito, jigyl).

12.-...AREIJES BORJA, vIRGILIO.-II:stor da la Medicina en ef Ecuador. Tdit, Cissia de lit C'utura Learoriala. 2 Vol. Quila, 11163 .

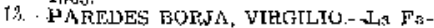

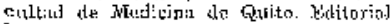
Jujverșitaria. Qequito, 1956. 